\title{
On the Manoussakis Conjecture for a Digraph to be Hamiltonian
}

\author{
Samvel Kh. Darbinyan \\ Institute for Informatics and Automation Problems of NAS RA \\ e-mail: samdarbin@ipia.sci.am
}

\begin{abstract}
Y. Manoussakis (J. Graph Theory 16, 1992, 51-59) proposed the following conjecture.

Conjecture. Let $D$ be a 2-strongly connected digraph of order $n$ such that for all distinct pairs of non-adjacent vertices $x, y$ and $w, z$, we have $d(x)+d(y)+d(w)+d(z) \geq$ $4 n-3$. Then $D$ is Hamiltonian.

In this note, we prove that if $D$ satisfies the conditions of this conjecture, then (i) $D$ has a cycle factor; (ii) If $\{x, y\}$ is a pair of non-adjacent vertices of $D$ such that $d(x)+d(y) \leq 2 n-2$, then $D$ is Hamiltonian if and only if $D$ contains a cycle passing through $x$ and $y$; (iii) If $\{x, y\}$ a pair of non-adjacent vertices of $D$ such that $d(x)+d(y) \leq 2 n-4$, then $D$ contains cycles of all lengths $3,4, \ldots, n-1$; (iv) $D$ contains a cycle of length at least $n-1$.
\end{abstract}

Keywords: Digraph, Hamiltonian cycle, Cycle factor, Pancyclic digraph.

\section{Introduction}

In this paper, we consider finite digraphs (directed graphs) without loops and multiple arcs. Every cycle and path are assumed simple and directed. A digraph $D$ is Hamiltonian if it contains a cycle passing through all the vertices of $D$. There are many conditions that guarantee that a digraph is Hamiltonian (see, e. g., [1]-[5]). In [5], the following theorem was proved.

Theorem 1.1: (Manoussakis [5]). Let D be a strongly connected digraph of order $n$. Suppose that $D$ satisfies the following condition for every triple $x, y, z \in V(D)$ such that $x$ and $y$ are non-adjacent: If there is no arc from $x$ to $z$, then $d(x)+d(y)+d^{+}(x)+d^{-}(z) \geq 3 n-2$. If there is no arc from $z$ to $x$, then $d(x)+d(y)+d^{-}(x)+d^{+}(z) \geq 3 n-2$. Then $D$ is Hamiltonian.

Definition 1.2: Let $D$ be a digraph of order $n$. We say that $D$ satisfies condition $(M)$ when $d(x)+d(y)+d(w)+d(z) \geq 4 n-3$ for all distinct pairs of non-adjacent vertices $x, y$ and $w, z$. 
Manoussakis [5] proposed the following conjecture. This conjecture is an extension of Theorem 1.1

Conjecture 1.3: (Manoussakis [5]). Let $G$ be a 2-strongly connected digraph of order $n$ such that for all distinct pairs of non-adjacent vertices $x, y$ and $w, z$ we have $d(x)+d(y)+d(w)+d(z) \geq 4 n-3$. Then $D$ is Hamiltonian.

This conjecture seems quite difficult to prove. Manoussakis [5] gave an example, which showed that if the conjecture is true, then the minimum degree condition is sharp. Notice that another examples can be found in [6], where for any two integers $k \geq 2$ and $m \geq 1$, the author constructed a family of $k$-strongly connected digraphs of order $4 k+m$ with minimum degree $4 k+m-1$, which are not Hamiltonian. This result improves a conjecture of Thomassen [2] (Conjecture 1.4.1). Moreover, when $m=1$, then from these digraphs we can obtain $k$-strongly connected non-Hamiltonian digraphs of order $n=4 k+1$ with minimum degree equal to $n-1$ and the minimal semi-degrees equal to $(n-3) / 2$. Thus, if in Conjecture 1.3 we replace $4 n-4$ instead of $4 n-3$, then for every $n$ there are many digraphs of order $n$ with high connection and high semi-degrees, for which Conjecture 1.3 is not true.

The author [7] proved the following theorem.

Theorem 1.4: (Darbinyan [7]). Let $D$ be a strongly connected digraph of order $n \geq 3$. Suppose that $d(x)+d(y) \geq 2 n-1$ for every pair of non-adjacent vertices $x, y \in V(D) \backslash\{z\}$, where $z$ is some vertex of $V(D)$. Then either $D$ is Hamiltonian or contains a cycle of length $n-1$.

It is easy to see that if a digraph $D$ satisfies the condition $(M)$, then it contains at most one pair of non-adjacent vertices $x, y$ such that $d(x)+d(y) \leq 2 n-2$. From this and Theorem 1.4 immediately follows the following corollary.

Corollary 1.5: Let $G$ be a strongly connected digraph of order $n$ satisfying condition $(M)$. Then $D$ contains a cycle of length at least $n-1$ (in particular, $D$ contains a Hamiltonian path).

Corollary 1.5 was also later proved by Ning [8].

In this paper we investigate the properties those digraphs, which satisfy the condition of Conjecture 1.3. Let $D$ be a 2-strongly connected digraph of order $n$ satisfying the condition $(M)$ and let $\{x, y\}$ be a pair of non-adjacent vertices of $D$. In Section 4 we prove:

(i) $D$ has a cycle factor;

(ii) If $d(x)+d(y) \leq 2 n-2$, then $D$ is Hamiltonian if and only if $D$ contains a cycle passing through $x$ and $y$;

(iii) If $d(x)+d(y) \leq 2 n-4$, then $D$ contains cycles of all lengths $3,4, \ldots, n-1$;

(iv) Suppose that $x_{1} x_{2} \ldots x_{n-2} y x_{1}$ is a cycle of length $n-1$ passing through $y$ and avoiding $x$. If $d(x)+d(y) \leq 2 n-2$ and $x_{n-2} \rightarrow x \rightarrow x_{1}$, then $D$ is Hamiltonian. 


\section{Terminology and Notation}

In this paper we consider finite digraphs without loops and multiple arcs. We shall assume that the reader is familiar with the standard terminology on digraphs and refer to [1] for terminology and notations not discussed here. The vertex set and the arc set of a digraph $D$ are denoted by $V(D)$ and $A(D)$, respectively. The order of $D$ is the number of its vertices. For any $x, y \in V(D)$, we also write $x \rightarrow y$ if $x y \in A(D)$. If $x y \in A(D), y$ is an out-neighbour of $x$ and $x$ is an in-neighbour of $y$. If $x \rightarrow y$ and $y \rightarrow z$, we write $x \rightarrow y \rightarrow z$. Two distinct vertices $x$ and $y$ are adjacent if $x y \in A(D)$ or $y x \in A(D)$ (or both). If there is no arc from $x$ to $y$, we shall use the notation $x y \notin A(D)$.

We let $N^{+}(x), N^{-}(x)$ denote the set of out-neighbours, respectively the set of inneighbours of a vertex $x$ in a digraph $D$. If $A \subseteq V(D)$, then $N^{+}(x, A)=A \cap N^{+}(x)$ and $N^{-}(x, A)=A \cap N^{-}(x)$. The out-degree of $x$ is $d^{+}(x)=\left|N^{+}(x)\right|$ and $d^{-}(x)=\left|N^{-}(x)\right|$ is the in-degree of $x$. Similarly, $d^{+}(x, A)=\left|N^{+}(x, A)\right|$ and $d^{-}(x, A)=\left|N^{-}(x, A)\right|$. The degree of the vertex $x$ in $D$ is defined as $d(x)=d^{+}(x)+d^{-}(x)$ (similarly, $d(x, A)=d^{+}(x, A)+d^{-}(x, A)$ ). The subdigraph of $D$ induced by a subset $A$ of $V(D)$ is denoted by $D\langle A\rangle$. If $z$ is a vertex of a digraph $D$, then the subdigraph $D\langle V(D) \backslash\{z\}\rangle$ is denoted by $D-z$.

For integers $a$ and $b, a \leq b$, let $[a, b]$ denote the set of all integers, which are not less than $a$ and are not greater than $b$.

The path (respectively, the cycle) consisting of the distinct vertices $x_{1}, x_{2}, \ldots, x_{m}(m \geq$ 2 ) and the $\operatorname{arcs} x_{i} x_{i+1}, i \in[1, m-1]$ (respectively, $x_{i} x_{i+1}, i \in[1, m-1]$, and $x_{m} x_{1}$ ), is denoted by $x_{1} x_{2} \cdots x_{m}$ (respectively, $x_{1} x_{2} \cdots x_{m} x_{1}$ ). The length of a cycle or path is the number of its arcs. We say that $x_{1} x_{2} \cdots x_{m}$ is a path from $x_{1}$ to $x_{m}$ or is an $\left(x_{1}, x_{m}\right)$-path. Let $x$ and $y$ be two distinct vertices of a digraph $D$. Cycle that passing through $x$ and $y$ in $D$, we denote by $C(x, y)$.

A cycle (respectively, a path) that contains all the vertices of $D$, is a Hamiltonian cycle (respectively, is a Hamiltonian path). A digraph is Hamiltonian if it contains a Hamiltonian cycle. A digraph $D$ of order $n \geq 3$ is pancyclic if it contains cycles of all lengths $m$, $3 \leq m \leq n$. For a cycle $C=x_{1} x_{2} \cdots x_{k} x_{1}$ of length $k$, the subscripts considered modulo $k$, i.e., $x_{i}=x_{s}$ for every $s$ and $i$ such that $i \equiv s(\bmod k)$. If $P$ is a path containing a subpath from $x$ to $y$, we let $P[x, y]$ denote that subpath. Similarly, if $C$ is a cycle containing vertices $x$ and $y, C[x, y]$ denotes the subpath of $C$ from $x$ to $y$.

A digraph $D$ is strongly connected, if there exists a path from $x$ to $y$ and a path from $y$ to $x$ for every pair of distinct vertices $x, y$. A digraph $D$ is $k$-strongly $(k \geq 1)$ connected if $|V(D)| \geq k+1$ and $D\langle V(D) \backslash A\rangle$ is strongly connected for any subset $A \subset V(D)$ of at most $k-1$ vertices.

Let $H$ be a non-trivial proper subdigraph of a digraph $D$. For the subdigraph $H$, a $H$ bypass is a path of length at least two with both end-vertices in $H$ and no other vertices in $H$. If $C$ is a non-Hamiltonian cycle in $D$ and $(x, y)$-path $P$ is a $C$-bypass with $V(P) \cap V(C)=$ $\{x, y\}$, then we call the length of the path $C[x, y]$ the gap of $P$ with respect to $C$.

A cycle factor in $D$ is a collection of vertex disjoint cycles $C_{1}, \ldots, C_{l}$ such that $V\left(C_{1}\right) \cup$ $\ldots \cup V\left(C_{l}\right)=V(D)$.

For a pair of disjoint subsets $A$ and $B$ of $V(D)$, we define $A(A \rightarrow B)=\{x y \in A(D) \mid x \in$ $A, y \in B\}$ and $A(A, B)=A(A \rightarrow B) \cup A(B \rightarrow A)$. 


\section{Preliminaries}

Lemma 3.1: (Häggkvist, Thomassen [9]). Let $D$ be a digraph of ordere $n \geq 3$ containing a cycle $C$ of length $m, m \in[2, n-1]$. Let $x$ be a vertex not contained in this cycle. If $d(x, V(C)) \geq m+1$, then $D$ contains a cycle of length $k$ for all $k \in[2, m+1]$.

The following lemma is a modification of a lemma by Bondy and Thomassen [10], its proof is almost the same.

Lemma 3.2: Let $D$ be a digraph of order $n \geq 3$ containing a path $P:=x_{1} x_{2} \ldots x_{m}$, $m \in[2, n-1]$. Let $x$ be a vertex not contained in this path. If one of the following statements holds:

(i) $d(x, V(P)) \geq m+2$;

(ii) $d(x, V(P)) \geq m+1$ and $x x_{1} \notin A(D)$ or $x_{m} x \notin A(D)$;

(iii) $d(x, V(P)) \geq m, x x_{1} \notin A(D)$ and $x_{m} x \notin A(D)$;

then there is an $i \in[1, m-1]$ such that $x_{i} x, x x_{i+1} \in A(D)$, i.e., $D$ contains a path $x_{1} x_{2} \ldots x_{i} x x_{i+1} \ldots x_{m}$ of length $m$ (we say that $x$ can be inserted into $P$ or the path $x_{1} x_{2} \ldots x_{i} x x_{i+1} \ldots x_{m}$ is an extended path obtained from $P$ with $\left.x\right)$.

It is not difficult to prove the following lemma.

Lemma 3.3: Let $D$ be a digraph of order $n$. Assume that $x y \notin A(D)$ and the vertices $x, y$ in $D$ satisfy the degree condition $d^{+}(x)+d^{-}(y) \geq n-2+k$, where $k \geq 1$. Then $D$ contains at least $k$ internally disjoint $(x, y)$-paths of length two.

Lemma 3.4: (Bypass Lemma, Bondy [11]). Let D be a strongly connected non-separable (i.e., $U G(D)$ is 2-connected) digraph and let $H$ be a non-trivial proper subdigraph of $D$. Then $D$ contains a H-bypass.

Theorem 3.4: (Yeo [12]). Let $D$ be a digraph. Then $D$ has a cycle factor if and only if $V(D)$ cannot be partitioned into subsets $Y, Z, R_{1}, R_{2}$ such that $A\left(Y \rightarrow R_{1}\right)=A\left(R_{2} \rightarrow R_{1} \cup Y\right)=\emptyset$, $|Y|>|Z|$ and $Y$ is an independent set.

Theorem 3.5: (Meyniel [4]). Let $D$ be a strongly connected digraph of order $n \geq 2$. If $d(x)+d(y) \geq 2 n-1$ for all pairs of non-adjacent vertices in $D$, then $D$ is Hamiltonian.

Before stating the main result of [14], we need to define a family of digraphs.

Definition 3.6: For any integers $n$ and $m,(n+1) / 2<m \leq n-1$, let $\Phi_{n}^{m}$ denote the set of digraphs $D$, which satisfy the following conditions: (i) $V(D)=\left\{x_{1}, x_{2}, \ldots, x_{n}\right\}$; (ii) $x_{n} x_{n-1} \ldots x_{2} x_{1} x_{n}$ is a Hamiltonian cycle in $D$; (iii) for each $k, 1 \leq k \leq n-m+1$, the vertices $x_{k}$ and $x_{k+m-1}$ are not adjacent; (iv) $x_{j} x_{i} \notin A(D)$ whenever $2 \leq i+1<j \leq n$ and (v) the sum of degrees for any two distinct non-adjacent vertices at least $2 n-1$.

Theorem 3.7: (Darbinyan [13], [14]). Let D be a strongly connected digraph of order $n \geq 3$. Suppose that $d(x)+d(y) \geq 2 n-1$ for all pairs of distinct non-adjacent vertices $x, y$ in $D$. Then either (a) $D$ is pancyclic or (b) $n$ is even and $D$ is isomorphic to one of $K_{n / 2, n / 2}^{*}$, 
$K_{n / 2, n / 2}^{*} \backslash\{e\}$, where $e$ is an arbitrary arc of $K_{n / 2, n / 2}^{*}$, or $(c) D \in \Phi_{n}^{m}$ (in this case $D$ does not contain a cycle of length $m$ ).

Later on, Theorem 3.7 was also proved by Benhocine [15].

\section{Proofs of the Results}

From the definition of condition $(M)$ the following lemma follows.

Lemma 4.1: Let $D$ be a digraph of order $n$ satisfying condition $(M)$. Then $D$ contains at most one pair of non-adjacent vertices $x$, y such that $d(x)+d(y) \leq 2 n-2$.

Theorem 4.2: Let $D$ be a 2-strongly connected digraph of order $n \geq 3$ satisfying condition $(M)$. Suppose that $\{x, y\}$ is a pair of non-adjacent vertices of $D$ such that $d(x)+d(y) \leq$ $2 n-2$. Then $D$ is Hamiltonian if and only if $D$ contains a cycle passing through the vertices $x$ and $y$.

Proof. If $D$ is Hamiltonian, then obviously it contain a cycle passing through $x$ and $y$. Suppose that $D$ contains a cycle passing through the vertices $x$ and $y$ but $D$ is not Hamiltonian. Let $C$ be a longest cycle, say of length $m$, passing through $x$ and $y$. Since $D$ is not Hamiltonian, we have that $m \leq n-1$. From 2-connectednees of $D$ and BypassLemma it follows that there is a $C$-bypass, say $P=u y_{1} y_{2} \ldots y_{k} v$, where $u, v \in V(C)$ and $y_{1}, y_{2}, \ldots, y_{k} \in V(D) \backslash V(C)$. Without loss of generality, assume that the gap $|C[u, v]|-1$ of $P$ is the minimum among the gaps of all $C$-bypasses. Then

$$
A\left(V(C[u, v]) \backslash\{u, v\}, V\left(P\left[y_{1}, y_{k}\right]\right)\right)=\emptyset .
$$

Put $f:=|V(C[u, v]) \backslash\{u, v\}|$. Since $C$ is a longest cycle passing through $x$ and $y$, it follows that $f \geq 1$. Now we extend the path $C[v, u]$ with the vertices of $V(C[u, v]) \backslash\{u, v\}$ as mach as possible. We obtain a $(v, u)$-path, say $R$. Then, since $C$ is a longest cycle passing through $x$ and $y, R$ does not contain some vertices $u_{1}, u_{2}, \ldots, u_{d}$ of $V(C[u, v]) \backslash\{u, v\}$. Using (1) and Lemma 3.2(i), for all $y_{j}$ and $u_{i}$ we obtain

$d\left(y_{j}, V(C)\right) \leq m-f+1 \quad$ and $\quad d\left(u_{i}, V(C)\right)=d\left(u_{i}, V(R)\right)+d\left(u_{i},\left\{u_{1}, \ldots, u_{d}\right\}\right) \leq m+d-1$.

By the minimality of the gap $f+1$ we also have that $D$ contains no path of the form $y_{j} z u_{i}$ and $u_{i} z y_{j}$, where $z \in B:=V(D) \backslash\left(V(C) \cup\left\{y_{1}, \ldots, y_{k}\right\}\right)$. Therefore,

$$
d\left(u_{i}, B\right)+d\left(y_{j}, B\right) \leq 2|B| .
$$

Now by a simple calculation we obtain

$$
\begin{gathered}
d\left(u_{i}\right)+d\left(y_{j}\right)=d\left(u_{i}, V(C)\right)+d\left(y_{j}, V(C)\right)+d\left(u_{i}, B\right)+d\left(y_{j}, B\right)+d\left(u_{i}, P\left[y_{1}, y_{k}\right]\right)+d\left(y_{j}, P\left[y_{1}, y_{k}\right]\right) \\
\leq m+d-1+m-f+1+2|B|+2 k-2 \leq 2 n-2,
\end{gathered}
$$

a contradiction with Lemma 4.1 since $u_{i}$ and $y_{j}$ are not adjacent and $\left\{u_{i}, y_{j}\right\} \neq\{x, y\}$. Theorem 4.2 is proved. 
Clearly, the existence of a cycle factor is a necessary condition for a digraph to be Hamiltonian.

Theorem 4.3: Let $D$ be a 2-strongly connected digraph of order $n$ satisfying condition $(M)$. Then $D$ has a cycle factor.

Proof. Suppose, on the contrary, that $D$ has no cycle factor. By the Yeo theorem, $V(D)$ can be partitioned into subsets $Y, Z, R_{1}, R_{2}$ such that $A\left(Y \rightarrow R_{1}\right)=A\left(R_{2} \rightarrow R_{1} \cup Y\right)=\emptyset$, $|Y|>|Z|$ and $Y$ is an independent set. Using these and 2-connectedness of $D$, we obtain that it follows that $|Z| \geq 2$ and hence, $|Y| \geq 3$. Let $x, y, z$ be three distinct vertices of $Y$. Since $Y$ is an independent set, we have that $\{x, y\}$ and $\{x, z\}$ are two distinct pairs of non-adjacent vertices of $V(D)$. Now using condition $(M)$, we obtain

$4 n-3 \leq 2 d(x)+d(y)+d(z) \leq 8|Z|+4\left|R_{1}\right|+4\left|R_{2}\right|+4|Y|-4|Y|=4 n-4(|Y|-|Z|) \leq 4 n-4$,

a contradiction. Theorem 4.3 is proved.

Using Yeo's theorem, it is not difficult to show that in Theorem 4.3 the minimum degree condition is sharp.

Theorem 4.4: Let $D$ be a 2-strongly connected digraph of order $n \geq 3$. Suppose that $D$ contains at most one pair of non-adjacent vertices. Then $D$ is Hamiltonian.

Proof. Suppose, on the contrary, that $D$ is not Hamiltonian. Therefore, $D$ is not semicomplete and contains exactly one pair, say $\{x, y\}$, of non-adjacent vertices. Then $d(x) \geq n-2$, $d(y) \geq n-2$ and $d(z) \geq n-1$ for all $z \in V(D)-\{x, y\}$. Since $D$ is 2-strongly connected, it follows that both subdigraphs $D-x$ and $D-y$ both are strongly connected semicomplete digraphs. Therefore, $D-x$ and $D-y$ both are Hamiltonian. Let $C_{n-1}:=x_{1} x_{2} \ldots x_{n-2} y x_{1}$ be a Hamiltonian cycle in $D-x$. Since $D$ is not Hamiltonian, from $d(x) \geq n-2$ and the fact that $x$ is adjacent to every vertex of $V(D)-\{y\}$ it follows that there exists an integer $l \in[2, n-3]$ such that

$$
\left\{x_{l+1}, x_{l+2}, \ldots, x_{n-2}\right\} \rightarrow x \rightarrow\left\{x_{1}, x_{2}, \ldots, x_{l}\right\} .
$$

Similarly, for some $k \in[2, n-3]$ we have

$$
\left\{x_{k+1}, x_{k+2}, \ldots, x_{n-2}\right\} \rightarrow y \rightarrow\left\{x_{1}, x_{2}, \ldots, x_{k}\right\} .
$$

Observe that for every pair of integers $i, j, 1 \leq i<j \leq n-2$, in $D$ there is no path of the types $x_{i} \rightarrow y \rightarrow x_{j}$ and $x_{i} \rightarrow z \rightarrow x_{j}$. By symmetry between $x$ and $y$, we may assume that $k \geq l$. Then by (2), (3) and our observation we have

$$
A\left(\{x, y\} \rightarrow\left\{x_{k+1}, x_{k+2}, \ldots, x_{n-2}\right\}\right)=\emptyset \quad \text { and } \quad\left\{x_{k+1}, x_{k+2}, \ldots, x_{n-2}\right\} \rightarrow\{x, y\} .
$$

From (4) and 2-connectedness of $D$ it follows that there exist $i \in[1, k-1]$ and $j \in[k+1, n-2]$ such that $x_{i} \rightarrow x_{j}$ (for otherwise, $A\left(\left\{x_{1}, x_{2}, \ldots, x_{k-1}, x, y\right\} \rightarrow\left\{x_{k+1}, x_{k+2}, \ldots, x_{n-2}\right\}\right)=\emptyset$, which means that $D-x_{k}$ is not strongly connected). Note that $y \rightarrow x_{i+1}$. We choose $j$ maximal with these properties. Using (2) and (3), it is easy to see that if $x_{j-1} \rightarrow x$, then $x_{1} \ldots x_{i} x_{j} \ldots x_{n-2} y x_{i+1} \ldots x_{j-1} x x_{1}$ is a Hamiltonian cycle, which contradicts our supposition. We may therefore assume that $x_{j-1} x \notin A(D)$. Then from $k \geq l$, (4) and 
the maximality of $j$ it follows that $j=k+1$ and $x_{k} x \notin A(D)$. Hence, $l=k$ and $A\left(\left\{x_{1}, x_{2}, \ldots, x_{k-1}\right\} \rightarrow\left\{x_{k+2}, x_{k+3}, \ldots, x_{n-2}\right\}\right)=\emptyset$. This together with (4) and 2connectedness of $D$ implies that there is an integer $s \in[k+2, n-2]$ such that $x_{k} \rightarrow x_{s}$. Therefore, $x_{1} \ldots x_{i} x_{k+1} \ldots x_{s-1} x x_{i+1} \ldots x_{k} x_{s} \ldots x_{n-2} y x_{1}$ is a Hamiltonian cycle, a contradiction. Theorem 4.4 is proved.

Remark: There is a strongly connected non-Hamiltonian digraph of order $n \geq 5$, which is not 2-strongly connected and has exactly one pair of non-adjacent vertices.

To see this, consider the following digraph $D$ defined as follows: $V(D)=\left\{x_{1}, x_{2}, \ldots, x_{n-2}, y, z\right\}, x_{1} x_{2}, \ldots x_{n-2} y x_{1}$ is a cycle of length $n-1$ in $D$,

$$
N^{-}(y)=N^{-}(z)=\left\{x_{k}, x_{k+1}, \ldots, x_{n-2}\right\} \quad \text { and } \quad N^{+}(y)=N^{+}(z)=\left\{x_{1}, x_{2}, \ldots, x_{k}\right\},
$$

where $k \in[2, n-3], D$ also contains all the $\operatorname{arcs} x_{j} x_{i}$ whenever $1 \leq i<j \leq n-2$ and it contain no other arcs.

It is not difficult to check that $D$ is neither 2-strongly connected nor Hamiltonian.

Lemma 4.5: Let $D$ be a 2-strongly connected digraph of order $n \geq 3$ and let $u$, $v$ be two distinct vertices in $D$. If $D$ contains no cycle passing through $u$ and $v$, then $u, v$ are not adjacent and there is no path of length two between them. In particular,

$$
d^{+}(u)+d^{-}(v) \leq n-2, \quad d^{-}(u)+d^{+}(v) \leq n-2 \quad \text { and } \quad d(u)+d(v) \leq 2 n-4 .
$$

Proof. It is obvious that $u, v$ are not adjacent. Suppose, on the contrary, that in $D$ there is a path of length two between the vertices $u$ and $v$, say $u \rightarrow z \rightarrow v$ or $v \rightarrow z \rightarrow u$. Since $D$ is 2-strongly connected, it follows that $D-z$ is strongly connected. Therefore, in $D-z$ there is an $(u, v)$ - and a $(v, u)$-path. It is easy to see that this $(u, v)$-path $((v, u)$-path, respectively) together with $v \rightarrow z \rightarrow u(u \rightarrow z \rightarrow v$, respectively) forms a cycle passing through $u$ and $v$. In both cases we have a contradiction, which proves that there is no path of length two between $u$ and $v$. Therefore, by Lemma 3.3, $d^{+}(u)+d^{-}(v) \leq n-2$ and $d^{-}(u)+d^{+}(v) \leq n-2$. These imply that $d(u)+d(v) \leq 2 n-4$. Lemma 4.5 is proved.

Theorem 4.6: Let $D$ be a 2-strongly connected digraph of order $n \geq 3$ satisfying condition $(M)$. Suppose that $\{u, v\}$ is a pair of non-adjacent vertices in $D$ such that $d(u)+d(v) \leq$ $2 n-2$. Then $D$ is Hamiltonian or $D$ contains a cycle of length $n-1$ passing through $u$ and avoiding $v$ (passing through $v$ and avoiding $u$ ).

Proof. Suppose that $D$ is not Hamiltonian. From Theorem 4.2 it follows that $D$ contains no cycle passing through $u$ and $v$. Therefore, by Lemma 4.5, $d(u)+d(v) \leq 2 n-4$. Since $D$ is 2-strongly connected, it follows that $D-u$ and $D-v$ both are strongly connected. From the last inequality and condition $(M)$ it follows that if $\{x, y\}$ is a pair of non-adjacent vertices in $D-u$ (in $D-v$, respectively), then the following inequalities holds:

$$
\begin{gathered}
d(x, V(D) \backslash\{u\})+d(y, V(D) \backslash\{u\}) \geq 2(n-1)-1, \\
(d(x, V(D) \backslash\{v\})+d(y, V(D) \backslash\{v\}) \geq 2(n-1)-1, \quad \text { respectively }) .
\end{gathered}
$$

Therefore, since $D-u$ and $D-v$ both are strongly connected, by Meyniel's theorem $D-u$ and $D-v$ both are Hamiltonian, i.e., $D$ contains a cycle of length $n-1$ passing through $u$ 
and avoiding $v$ (passing through $v$ and avoiding $u$ ). Theorem 4.6 is proved.

As an immediate corollary of Theorems 4.2 and 4.6 (respectively, Theorem 4.6 and Corollary 3.1), we obtain Corollary 4.7 (respectively, Corollary 4.8).

Corollary 4.7: Let $D$ be a 2-strongly connected non-Hamiltonian digraph of order $n \geq 3$ satisfying condition $(M)$. Suppose that $\{u, v\}$ is a pair of non-adjacent vertices in $D$ such that $d(u)+d(v) \leq 2 n-2$. Then $D$ contains at most one cycle of length two passing through $u(v)$.

Corollary 4.8: Let $D$ be a 2-strongly connected non-Hamiltonian digraph of order $n \geq 3$ satisfying condition $(M)$. Suppose that $\{u, v\}$ is a pair of non-adjacent vertices in $D$ such that $d(u)+d(v) \leq 2 n-2$. Then $d(u) \leq n-1$ and $d(v) \leq n-1$.

Theorem 4.9: Let $D$ be a 2-strongly connected digraph of order $n \geq 6$ satisfying condition $(M)$. Suppose that $\{x, y\}$ is a pair of non-adjacent vertices in $D$ such that $d(x)+d(y) \leq$ $2 n-4$. Then $D$ contains cycles of all lengths $3,4, \ldots, n-1$.

Proof. Suppose first that $D$ contains exactly one pair of non-adjacent vertices, namely $\{x, y\}$. Then $D-x$ is a strongly connected semicomplete digraph. Therefore, by the wellknown theorem of Moser [16], $D-x$ contains cycles of all lengths $3,4, \ldots, n-1$.

Suppose next that $D$ contains at least two distinct pairs of non-adjacent vertices. Let $\{u, v\}$ be an arbitrary pair of non-adjacent vertices in $V(D) \backslash\{x\}$ (or in $V(D) \backslash\{y\}$ ). From condition $(M)$ it follows that

$$
d(u)+d(v) \geq 2 n+1 .
$$

Now we consider the subdigraph $H:=D-x$. For the digraph $H$ we first prove the following claim.

Claim: If $H \cong K_{m, m}^{*}-e$, where $e$ is an arbitrary arc of $K_{m, m}^{*}$, then $D$ contains cycles of all lengths $2,3, \ldots, n-1$.

Proof. Let $\{u, v\}$ be an arbitrary pair of non-adjacent vertices in $K_{m, m}^{*}-e$. Note that $n=2 m+1 \geq 7$. Then

$$
d(u, V(D) \backslash\{x\})+d(v, V(D) \backslash\{x\}) \leq 4 m=2 n-2 .
$$

Therefore, by (5), $d(x,\{u, v\}) \geq 3$. This, since $m \geq 3$, in turn, implies that every partite set of $H$ contains at least two vertices such that each of them together with $x$ forms a 2-cycle. Therefore, there exist two vertices $z, w \in V(H)$ such that $z \leftrightarrow w, z \leftrightarrow x$ and $w \leftrightarrow x$ Then, since for every $k, k \in[1, m]$ there is a cycle of length $2 k$ passing through the arc $z \rightarrow w$, it follows that $D$ contains cycles of all lengths $2,3, \ldots, n$. The claim is proved.

We now return to the proof of Theorem 4.9. From (5) it also follows that

$$
d(u, V(D) \backslash\{x\})+d(v, V(D) \backslash\{x\}) \geq 2(n-1)-1 .
$$

Then, since $H$ is strongly connected, from Theorem 3.7 it follows that either $H$ contains cycles of all lengths $3,4, \ldots, n-1$ or $H \in\left\{K_{m, m}^{*}, K_{m, m}^{*}-e\right\} \cup \Phi_{n-1}^{k}$, where $n / 2<k \leq n-2$. In order to complete the proof of the theorem, by the above claim it suffices to consider only 
the case when $H \in \Phi_{n-1}^{k}$. From the definition of the set $\Phi_{n-1}^{k}$ it follows that $H$ contains cycles of all lengths $2,3, \ldots, n-1$ except the cycle of length $k$.

Let $x_{1} x_{n-1} x_{n-2} \ldots x_{2} x_{1}$ be a Hamiltonian cycle in $H$. Since $H \in \Phi_{n-1}^{k}$, it follows that $\left\{x_{1}, x_{k}\right\},\left\{x_{n-k}, x_{n-1}\right\}$ are two distinct pairs of non-adjacent vertices other than $\{x, y\}$ and

$$
d\left(x_{1}, V(H)\right)+d\left(x_{k}, V(H)\right)=d\left(x_{n-k}, V(H)\right)+d\left(x_{n-1}, V(H)\right)=2 n-3 .
$$

This together with (5) implies that $d\left(x,\left\{x_{1}, x_{n-k}, x_{k}, x_{n-1}\right)=8\right.$. If $k \neq n-3$, then $x_{1} \rightarrow x_{n-3}$ and $x_{1} x_{n-3} x_{n-4} \ldots x_{n-k} x x_{1}$ is a cycle of length $k$. Assume that $k=n-3$. Then $\left\{x_{1}, x_{n-3}\right\},\left\{x_{3}, x_{n-1}\right\}$ are two pairs of non-adjacent vertices other than $\{x, y\}$. We have that $d\left(x,\left\{x_{1}, x_{3}, x_{n-3}, x_{n-1}\right)=8, x_{1} \rightarrow x_{n-4}\right.$ and $x_{3} \rightarrow x_{n-1}$. If $x_{2} \rightarrow x$, then $x_{1} x_{n-4} \ldots x_{3} x_{2} x x_{1}$ is a cycle of length $n-3$. Assume that $x_{2} x \notin A(D)$. Then, since the vertices $x_{2}$ and $x_{n-2}$ are not adjacent and $d\left(x_{2}\right)+d\left(x_{n-2}\right) \geq 2 n+1$, it is not difficult to see that $x_{2} \rightarrow x_{n-3}$ and $x \rightarrow x_{2}$. Therefore, $x x_{2} x_{n-3} x_{n-4} x_{3} x$ is a cycle of length $n-3$. This completes the proof of the theorem.

In view of Theorem 4.9, it is natural to set the following problem.

Problem: Let $D$ be a 2-strongly connected digraph of order $n$ satisfying condition $(M)$. Suppose that $\{x, y\}$ is a pair of non-adjacent vertices in $D$ such that $2 n-3 \leq d(x)+d(y) \leq 2 n-2$. Whether $D$ contains cycles of all lengths $3,4, \ldots, n-1$ ?

\section{Remarks}

In the following, we suppose, further, that $D$ is a 2-strongly connected digraph of order $n$ satisfying condition $(M)$. Moreover, $D$ contains a pair $\{y, z\}$ of non-adjacent distinct vertices $y, z$ such that $d(y)+d(z) \leq 2 n-4$. In this section, we will prove a number of properties of $D$.

Lemma 5.1: Let $x_{1} x_{2} \ldots x_{n-2} z x_{1}$ be a cycle of length $n-1$ in $D$, which does not contain $y$. Suppose that $x_{a} \rightarrow x_{b}, x_{q} \rightarrow y \rightarrow x_{p}$ and $x_{t} \rightarrow y \rightarrow x_{s}$, where $1 \leq s \leq a<p \leq q<b \leq$ $t \leq n-2$. Then $D$ is Hamiltonian.

Proof. Suppose, on the contrary, that $D$ is not Hamiltonian. By Theorem $4.2, D$ contains no cycle passing through $y$ and $z$. Notice that there are no integers $l$ and $r, 1 \leq l<r \leq n-2$, such that $x_{l} \rightarrow y \rightarrow x_{r}$ (for otherwise, $x_{1} \ldots x_{l} y x_{r} \ldots x_{n-2} z x_{1}$ is a cycle passing through $y$ and $z$. If $z \rightarrow x_{i}$ with $i \in[a+1, q]$, then $C(y, z)=x_{s} \ldots x_{a} x_{b} \ldots x_{n-2} z x_{i} \ldots x_{q} y x_{s}$;

if $x_{j} \rightarrow z$ with $j \in[p, b-1]$, then $C(y, z)=x_{1} \ldots x_{a} x_{b} \ldots x_{t} y x_{p} \ldots x_{j} z x_{1}$. Thus, in both cases we have a contradiction. Therefore,

$$
d^{+}\left(z,\left\{x_{a+1}, \ldots, x_{q}\right\}\right)=d^{-}\left(z,\left\{x_{p}, \ldots, x_{b-1}\right\}\right)=0
$$

in particular, $d\left(z,\left\{x_{p}, \ldots, x_{q}\right\}\right)=0$ and the vertices $z$ and $x_{p}$ are not adjacent. The last equality together with the fact that $D$ contains at most one cycle of length two passing through $z$ (Corollary 4.7) implies that

$$
d(z)=d\left(z,\left\{x_{1}, \ldots, x_{p-1}\right\}\right)+d\left(z,\left\{x_{q+1}, \ldots, x_{n-2}\right\}\right) \leq p-1+n-2-q+1=n+p-q-2 .
$$


Now we consider the vertex $x_{p}$. It is easy to see that if $x_{i} \rightarrow x_{p}$ with $i \in[1, s-1]$, then $C(y, z)=x_{1} \ldots x_{i} x_{p} \ldots x_{q} y x_{s} \ldots x_{a} x_{b} \ldots x_{n-2} z x_{1}$, if $x_{p} \rightarrow x_{j}$ with $j \in[t+1, n-2]$, then $C(y, z)=x_{1} \ldots x_{a} x_{b} \ldots x_{t} y x_{p} x_{j} \ldots x_{n-2} z x_{1}$. In both cases we have a contradiction. Therefore, we may assume that

$$
d^{-}\left(x_{p},\left\{x_{1}, \ldots, x_{s-1}\right\}\right)=d^{+}\left(x_{p},\left\{x_{t+1}, \ldots, x_{n-2}\right\}\right)=0 .
$$

This implies that

$$
\begin{gathered}
d\left(x_{p}\right)=d^{+}\left(x_{p},\left\{x_{1}, \ldots, x_{s-1}\right\}\right)+d^{-}\left(x_{p},\left\{x_{t+1}, \ldots, x_{n-2}\right\}\right)+d\left(x_{p},\left\{x_{s}, \ldots, x_{t}\right\}\right)+d\left(x_{p},\{y\}\right) \\
\leq s-1+n-2-t+2(t-s+1)=n+t-s-1 .
\end{gathered}
$$

Without loss of generality, we may assume that $s, q$ are chosen as maximal as possible and $p, t$ are chosen as minimal as possible. Then

$$
d\left(y,\left\{x_{s+1}, \ldots, x_{p-1}\right\}\right)=d\left(y,\left\{x_{q+1}, \ldots, x_{t-1}\right\}\right)=0 .
$$

This, since $D$ contains at most one cycle of length two passing through $y$, implies that

$$
\begin{gathered}
d(y)=d\left(y,\left\{x_{1}, \ldots, x_{s}\right\}\right)+d\left(y,\left\{x_{p}, \ldots, x_{q}\right\}\right)+d\left(y,\left\{x_{t}, \ldots, x_{n-2}\right\}\right) \\
\leq s+q-p+1+n-2-t+1+1=n+s+q-p-t+1 .
\end{gathered}
$$

Since $\{y, z\}$ and $\left\{x_{p}, z\right\}$ are two distinct pairs of non-adjacent vertices, from (6), (7), (8) and condition $(M)$ it follows that

$$
4 n-3 \leq d(y)+2 d(z)+d\left(x_{p}\right) \leq 4 n-4-(q-p) \leq 4 n-4,
$$

which is a contradiction. Lemma 5.1 is proved.

The following claim is an immediate consequence of Lemma 5.1.

Claim 1: Let $x_{1} x_{2} \ldots x_{n-2} z x_{1}$ be a cycle of length $n-1$ in $D$ passing through $z$. If $x_{n-2} \rightarrow y \rightarrow x_{1}$ and $x_{1} \rightarrow x_{n-2}$, then $D$ is Hamiltonian.

The following claim will be very useful in the remaining proof.

Claim 2: Let $x_{1} x_{2} \ldots x_{n-2}$ be a Hamiltonian path in $D-\{y, z\}$. Suppose that for every pair of integers $i$ and $j, 1 \leq i<j \leq n-2$, if $x_{i} \rightarrow y$, then $y x_{j} \notin A(D)$, and if $x_{i} \rightarrow z$, then $z x_{j} \notin A(D)$. Then either $D$ is Hamiltonian or for every $k \in[2, n-3]$, the following holds:

$$
A\left(\left\{x_{1}, x_{2}, \ldots, x_{k-1}\right\} \rightarrow\left\{x_{k+1}, x_{k+2}, \ldots, x_{n-2}\right\}\right) \neq \emptyset .
$$

Proof. Suppose, on the contrary, that $D$ is not Hamiltonian and there is an integer $k \in$ $[2, n-3]$ such that

$$
A\left(\left\{x_{1}, x_{2}, \ldots, x_{k-1}\right\} \rightarrow\left\{x_{k+1}, x_{k+2}, \ldots, x_{n-2}\right\}\right)=\emptyset,
$$

We can assume that the vertices $x_{m}$ and $x_{l}$ are chosen so that $y \rightarrow x_{m}, z \rightarrow x_{l}$ and

$$
d^{+}\left(y,\left\{x_{m+1}, \ldots, x_{n-2}\right\}\right)=d^{+}\left(z,\left\{x_{l+1}, \ldots, x_{n-2}\right\}\right)=0 .
$$


Without loss of generality, we assume that $m \leq l$. Since $D$ is 2 -strongly connected, it follows that $2 \leq m \leq l \leq n-3$. From the supposition of this claim and (9) it follows that:

(i) if $k \leq m$ or $k \geq l$, then (respectively)

$$
A\left(\left\{x_{1}, x_{2}, \ldots, x_{k-1}\right\} \rightarrow\left\{y, z, x_{k+1}, x_{k+2}, \ldots, x_{n-2}\right\}\right)=\emptyset
$$

or

$$
A\left(\left\{y, z, x_{1}, x_{2}, \ldots, x_{k-1}\right\} \rightarrow\left\{x_{k+1}, x_{k+2}, \ldots, x_{n-2}\right\}\right)=\emptyset,
$$

(ii) if $m+1 \leq k \leq l-1$, then

$$
A\left(\left\{y, x_{1}, x_{2}, \ldots, x_{k-1}\right\} \rightarrow\left\{z, x_{k+1}, x_{k+2}, \ldots, x_{n-2}\right\}\right)=\emptyset .
$$

Thus, in each case we have that $D-x_{k}$ is not strongly connected, which contradicts that $D$ is 2-strongly connected. Claim 2 is proved.

Lemma 5.2: Suppose that $x_{1} x_{2} \ldots x_{n-2} z x_{1}$ is a cycle in $D$ passing through $z$ and avoiding $y$. If $x_{n-2} \rightarrow y \rightarrow x_{1}$, then $D$ is Hamiltonian.

Proof. Suppose, on the contrary, that $x_{n-2} \rightarrow y \rightarrow x_{1}$ but $D$ is not Hamiltonian. By Theorem 4.2, $D$ contains no cycle passing through $y$ and $z$ in $D$. It is easy to see that the conditions of Claim 2 hold. Let $x_{k} \rightarrow y \rightarrow x_{p}$, where $2 \leq p \leq k \leq n-3, k$ minimal and $p$ maximal with this property. Since $D$ is 2 -strongly connected, from Lemma 5.1 it follows that $p \leq k-1$. This means that there is no cycle of length two passing through $y$. By symmetry between the vertices $y$ and $z$, we may assume that also there is no cycle of length two passing through $z$.

Case 1. $p=k-1$. By Lemma 5.1 we have that

$$
A\left(\left\{x_{1}, \ldots, x_{p-1}\right\} \rightarrow\left\{x_{k+1}, \ldots, x_{n-2}\right\}\right)=\emptyset .
$$

Then, by Claim 2, there are some integers $i \in[1, p-1]$ and $j \in[k+1, n-2]$ such that $x_{i} \rightarrow x_{k}$ and $x_{k-1} \rightarrow x_{j}$. Therefore, $C(y, z)=x_{1} \ldots x_{i} x_{k} y x_{k-1} x_{j} \ldots x_{n-2} z x_{1}$ is a cycle passing through $y$ and $z$, which is a contradiction.

Case 2. $p \leq k-2$. Then $d\left(y,\left\{x_{p+1}, \ldots, x_{k-1}\right\}\right)=0$. By Lemma 5.1 we have that

$$
A\left(\left\{x_{1}, \ldots, x_{p-1}\right\} \rightarrow\left\{x_{k+1}, \ldots, x_{n-2}\right\}\right)=\emptyset .
$$

Therefore, by Claim 2, there are $s \in[1, p-1], a \in[p+1, k], b \in[p, k-1]$ and $t \in[k+1, n-2]$ such that $x_{s} \rightarrow x_{a}$ and $x_{b} \rightarrow x_{t}$. If $a>b$, then $x_{1} \ldots x_{s} x_{a} \ldots x_{k} y x_{p} \ldots x_{b} x_{t} \ldots x_{n-2} z x_{1}$ is a cycle passing through $y$ and $z$, which is a contradiction. We may therefore assume that $a \leq b$. Then $p+1 \leq a \leq b \leq k-1$ and the vertices $y$ and $x_{a}$ are not adjacent. Choose (i) $a$ and $t$ as maximal as possible and (ii) choose $b$ and $s$ as minimal as possible, subject to (i). This means that

$$
A\left(\left\{x_{1}, \ldots, x_{p-1}\right\} \rightarrow\left\{x_{a+1}, \ldots, x_{n-2}\right\}\right)=A\left(\left\{x_{1}, \ldots, x_{b-1}\right\} \rightarrow\left\{x_{k+1}, \ldots, x_{n-2}\right\}\right)=\emptyset .
$$

From the minimality of $s$ and the maximality of $t$ we have that

$$
\begin{gathered}
d\left(x_{a}\right)=d^{+}\left(x_{a},\left\{x_{1}, \ldots, x_{s-1}\right\}\right)+d^{-}\left(x_{a},\left\{x_{t+1}, \ldots, x_{n-2}\right\}\right)+d\left(x_{a},\left\{x_{s}, \ldots, x_{t}\right\}\right)+d\left(x_{a},\{z\}\right) \\
\leq s-1+n-2-t+2 t-2 s+1=n+t-s-2 .
\end{gathered}
$$


Let $m$ be the number of vertices of the set $\left\{x_{s+1}, \ldots, x_{p}, x_{k}, \ldots, x_{t-1}\right\}$, which are not adjacent to $y$. Then, since $y$ is not on the cycle of length two and $d\left(y,\left\{x_{p+1}, \ldots, x_{k-1}\right\}\right)=0$, it follows that

$$
d(y) \leq n-2-m-(k-1-p)=n+p-m-k-1 .
$$

Assume first that

$$
d^{+}\left(z,\left\{x_{s+1}, \ldots, x_{p}\right\}\right)=d^{-}\left(z,\left\{x_{k}, \ldots, x_{t-1}\right\}\right)=0 .
$$

From this and taking into account Lemma 4.5 (there is no path of length two between $y$ and $z$ ) we obtain that

$$
d(z) \leq s+n-2-t+1+m+k-1-p=n+k+m+s-t-p-2 .
$$

Combining (12)-(14), $k-p \geq 2$ and $m \geq 0$, we obtain

$$
2 d(y)+d\left(x_{a}\right)+d(z) \leq 4 n-6-(k-p)-m \leq 4 n-8,
$$

which is a contradiction to condition $(M)$, since $\{y, z\}$ and $\left\{y, x_{a}\right\}$ are two distinct pairs of non-adjacent vertices.

Assume next that

$$
d^{+}\left(z,\left\{x_{s+1}, \ldots, x_{p}\right\}\right) \neq 0 \quad \text { or } \quad d^{-}\left(z,\left\{x_{k}, \ldots, x_{t-1}\right\}\right) \neq 0,
$$

i.e., there is a $q \in[s+1, p]$ such that $z \rightarrow x_{q}$ or there is a $r \in[k, t-1]$ such that $x_{r} \rightarrow z$.

Using Claim 2, we obtain that $A\left(\left\{x_{1}, \ldots, x_{a-1}\right\} \rightarrow\left\{x_{a+1}, \ldots, x_{n-2}\right\}\right) \neq \emptyset$. Let $x_{s_{1}} \rightarrow x_{t_{1}}$, where $s_{1} \in[1, a-1]$ and $t_{1} \in[a+1, n-2]$. From (11) it follows that $s_{1} \in[p, a-1]$ and $t_{1} \in[a+1, k]$. Choose $t_{1}$ maximal with this property. Then

$$
A\left(\left\{x_{1}, \ldots, x_{a-1}\right\} \rightarrow\left\{x_{t_{1}+1}, \ldots, x_{n-2}\right\}\right)=\emptyset .
$$

Now using the facts that $z \rightarrow x_{q}$ or $x_{r} \rightarrow z$, it is not difficult to check that: if $t_{1}>b$, then $C(y, z)=x_{1} \ldots x_{s} x_{a} \ldots x_{b} x_{t} \ldots x_{n-2} z x_{q} \ldots x_{s_{1}} x_{t_{1}} \ldots x_{k} y x_{1}$ or $C(y, z)=$ $x_{1} \ldots x_{s} x_{a} \ldots x_{b} x_{t} \ldots x_{n-2} y x_{p} \ldots x_{s_{1}} x_{t_{1}} \ldots x_{r} z x_{1}$ is a cycle passing through $y$ and $z$, when $z \rightarrow x_{q}$ and $x_{r} \rightarrow z$, respectively. In both cases we have a contradiction. We may therefore assume that $t_{1} \leq b$.

From Claim 2 we have that $A\left(\left\{x_{1}, \ldots, x_{t_{1}-1}\right\} \rightarrow\left\{x_{t_{1}+1}, \ldots, x_{n-2}\right\}\right) \neq \emptyset$. Let $x_{s_{2}} \rightarrow x_{t_{2}}$, where $s_{2} \in\left[1, t_{1}-1\right]$ and $t_{2} \in\left[t_{1}+1, n-2\right]$. From (11) and (15) it follows that $s_{2} \in\left[a, t_{1}-1\right]$ and $t_{2} \in\left[t_{1}+1, k\right]$. Choose $t_{2}$ maximal with this property. Then

$$
A\left(\left\{x_{1}, \ldots, x_{t_{1}-1}\right\} \rightarrow\left\{x_{t_{2}+1}, \ldots, x_{n-2}\right\}\right)=\emptyset .
$$

If $t_{2}>b$, then $C(y, z)=x_{1} \ldots x_{s} x_{a} \ldots x_{s_{2}} x_{t_{2}} \ldots x_{k} y x_{p} \ldots x_{s_{1}} x_{t_{1}} \ldots x_{b} x_{t} \ldots x_{n-2} z x_{1}$, a contradiction. We may therefore assume that $t_{2} \leq b$. In particular, from $t_{2} \geq t_{1}+1$ it follows that $t_{1}<b$.

Using Claim 2, (11) and (16), we obtain that there are some integers $s_{3} \in\left[t_{1}, t_{2}-1\right]$ and $t_{3} \in\left[t_{2}+1, k\right]$ such that $x_{s_{3}} \rightarrow x_{t_{3}}$. Choose $t_{3}$ maximal with this properties. Then

$$
A\left(\left\{x_{1}, \ldots, x_{t_{2}-1}\right\} \rightarrow\left\{x_{t_{3}+1}, \ldots, x_{n-2}\right\}\right)=\emptyset .
$$


If $t_{3}>b$, then $C(y, z)=x_{1} \ldots x_{s} x_{a} \ldots x_{s_{2}} x_{t_{2}} \ldots x_{b} x_{t} \ldots x_{n-2} z x_{q} \ldots x_{s_{1}} x_{t_{1}} \ldots x_{s_{3}} x_{t_{3}} \ldots x_{k} y x_{1}$ or $C(y, z)=x_{1} \ldots x_{s} x_{a} \ldots x_{s_{2}} x_{t_{2}} \ldots x_{b} x_{t} \ldots x_{n-2} y x_{p} \ldots x_{s_{1}} x_{t_{1}} \ldots x_{s_{3}} x_{t_{3}} \ldots x_{r} z x_{1}$, when $z \rightarrow$ $x_{q}$ or $x_{r} \rightarrow z$, respectively. We may therefore assume that $t_{3} \leq b$. Then $t_{2}<b$.

Again using Claim 2, (11) and (17), we obtain that there are some integers $s_{4} \in$ $\left[t_{2}, t_{3}-1\right]$ and $t_{4} \in\left[t_{3}+1, k\right]$ such that $x_{s_{4}} \rightarrow x_{t_{4}}$. If $t_{4}>b$, then $C(x, y)=$ $x_{1} \ldots x_{s_{0}} x_{t_{0}} \ldots x_{s_{2}} x_{t_{2}} \ldots x_{s_{4}} x_{t_{4}} \ldots x_{k} y x_{p} \ldots x_{s_{1}} x_{t_{1}} \ldots x_{s_{3}} x_{t_{3}} \ldots x_{b} x_{t} \ldots x_{n-2} z x_{1}$, a contradiction. (Here, $x_{s}:=x_{s_{0}}$ and $x_{a}=x_{t_{0}}$ ).

Continuing this process, we finally conclude that for some $l \geq 0, t_{l}>b$ since all the vertices $x_{t_{0}}, x_{t_{1}}, \ldots, x_{t_{l}}$ are distinct and in $\left\{x_{p}, \ldots, x_{k}\right\}$. By the above arguments we have that: if $t_{l}$ is even, then $C(y, z)=x_{1} \ldots x_{s_{0}} x_{t_{0}} \ldots x_{s_{2}} x_{t_{2}} \ldots x_{s_{4}} x_{t_{4}} \ldots x_{s_{l}} x_{t_{l}} \ldots x_{k} y x_{p} \ldots x_{s_{1}} x_{t_{1}} \ldots$ $x_{s_{3}} x_{t_{3}} \ldots x_{s_{l-1}} x_{t_{l-1}} \ldots x_{b} x_{t} \ldots x_{n-2} z x_{1}$, if $l$ is odd, then $C(y, z)=x_{1} \ldots x_{s_{0}} x_{t_{0}} \ldots x_{s_{2}} x_{t_{2}} \ldots$ $x_{s_{l-1}} x_{t_{l-1}} \ldots x_{b} x_{t} \ldots x_{n-2} z x_{q} \ldots x_{s_{1}} x_{t_{1}} \ldots x_{s_{3}} x_{t_{3}} \ldots x_{s_{l}} x_{t_{l}} \ldots x_{k} y x_{1}$, or $C(y, z)=x_{1} \ldots x_{s_{0}} x_{t_{0}}$ $\ldots x_{s_{2}} x_{t_{2}} \ldots x_{s_{l-1}} x_{t_{l-1}} \ldots x_{b} x_{t} \ldots x_{n-2} y x_{p} \ldots x_{s_{1}} x_{t_{1}} \ldots x_{s_{3}} x_{t_{3}} \ldots x_{s_{l}} x_{t_{l}} \ldots x_{r} z x_{1}$, when $z \rightarrow x_{q}$ or $x_{r} \rightarrow z$, respectively. In all cases we have a cycle passing through $y$ and $z$, which contradicts our supposition. Lemma 5.2 is proved.

From Theorem 4.4, Lemma 5.2 and Corollary 4.7 the following corollary follows.

Corollary 5.3: If $D$ is not Hamiltonian, then $\max \{d(y), d(z)\} \leq n-2$.

Lemma 5.4: Let $C=x_{1} x_{2} \ldots x_{n-3} x_{1}$ be a cycle of length $n-3$ in $D$ passing through $y$ and avoiding z. Let $V(D) \backslash V(C)=\{z, u, v\}$. If

(i). $d(y,\{u, v\})=0$ and zuvz is a cycle of length 3 or

(ii). $d(y) \leq n-3$ and $z \leftrightarrow u$, then $D$ is Hamiltonian.

Proof. Suppose, on the contrary, that $D$ is not Hamiltonian. Then, by Theorem $4.2, D$ contains no cycle passing through $y$ and $z$.

(i). Since $d(y,\{z, u, v\})=0$ and through $y$ there is at most one cycle of length two, it follows that $d(y) \leq n-3$. It is easy to see that for every $i \in[1, n-3]$ the following holds:

$$
\vec{a}\left[x_{i}, z\right]+\vec{a}\left[v, x_{i+1}\right] \leq 1, \quad \vec{a}\left[x_{i}, u\right]+\vec{a}\left[z, x_{i+1}\right] \leq 1 \quad \text { and } \quad \vec{a}\left[x_{i}, v\right]+\vec{a}\left[u, x_{i+1}\right] \leq 1,
$$

(for otherwise, $D$ is Hamiltonian). Therefore,

$$
\begin{gathered}
d(z, V(C))+d(u, V(C))+d(v, V(C)) \\
=\sum_{i=1}^{n-3}\left(\vec{a}\left[x_{i}, z\right]+\vec{a}\left[v, x_{i+1}\right]+\vec{a}\left[x_{i}, u\right]+\vec{a}\left[z, x_{i+1}\right]+\vec{a}\left[x_{i}, v\right]+\vec{a}\left[u, x_{i+1}\right]\right) \leq 3 n-9 .
\end{gathered}
$$

Then, since $d(z,\{u, v\}) \leq 3$ and $d(u,\{z, v\})+d(v,\{u, z\}) \leq 7$ it follows that $d(z)+d(u)+$ $d(v) \leq 3 n+1$. Therefore, since $2 d(y)+d(z)+d(u) \geq 4 n-3$ and $d(v)+d(y) \geq 2 n+1$, we have

$$
6 n-2 \leq 3 d(y)+d(u)+d(v)+d(z) \leq 6 n-8,
$$

which is a contradiction.

(ii). Then for every $i \in[1, n-3]$ we have $\vec{a}\left[x_{i}, z\right]+\vec{a}\left[u, x_{i+1}\right] \leq 1$ and $\vec{a}\left[x_{i}, u\right]+$ $\vec{a}\left[z, x_{i+1}\right] \leq 1$. Therefore,

$$
d(z, V(C))+d(u, V(C))=\sum_{i=1}^{n-3}\left(\vec{a}\left[x_{i}, z\right]+\vec{a}\left[u, x_{i+1}\right]+\vec{a}\left[x_{i}, u\right]+\vec{a}\left[z, x_{i+1}\right]\right) \leq 2 n-6 .
$$


Hence, $d(z)+d(u) \leq 2 n+1$. This together with $d(y) \leq n-3$ and condition $(M)$ gives

$$
4 n-3 \leq d(z)+d(u)+2 d(y) \leq 2 n+1+2 n-6=2 n-5,
$$

which is a contradiction. Lemma 5.4 is proved.

Lemma 5.5: Let $C:=x_{1} x_{2} \ldots x_{n-4} x_{1}$ be a cycle of length $n-4$ in $D$ passing through $y$ and avoiding $z$. Let $V(D) \backslash V(C))=\left\{z, u_{1}, u_{2}, u_{3}\right\}$. If one of the following conditions holds

(i). $d(y) \leq n-3$ and $z \leftrightarrow u_{1}$,

ii). $z u_{1} u_{2} z$ is a cycle of length 3 and $d\left(y,\left\{u_{1}, u_{2}\right\}\right)=0$,

(iii). $z u_{1} u_{2} u_{3} z$ is a cycle of length 4 and $d\left(y,\left\{u_{1}, u_{2}, u_{3}\right\}\right)=0$, then $D$ is Hamiltonian.

Proof. Suppose, on the contrary, that $D$ is not Hamiltonian. By Theorem $4.2, D$ contains no cycle passing through $y$ and $z$.

(i). Note that $y$ and $u_{1}$ are not adjacent by Lemma 4.5. Since $z \leftrightarrow u_{1}$, it is easy to see that $\vec{a}\left[x_{i}, z\right]+\vec{a}\left[u_{1}, x_{i+1}\right] \leq 1$ and $\vec{a}\left[x_{i}, u_{1}\right]+\vec{a}\left[z, x_{i+1}\right] \leq 1$. Hence, $d(z, V(C))+$ $d\left(u_{1}, V(C)\right) \leq 2 n-8$. Therefore, since, $d\left(u_{1},\left\{z, u_{2}, u_{3}\right\}\right) \leq 6$ and $d\left(z,\left\{u_{1}, u_{2}, u_{3}\right\}\right) \leq 4$, we have $d(z)+d\left(u_{1}\right) \leq 2 n+2$. This together with $d(y) \leq n-3$ and condition $(M)$ implies that

$$
4 n-3 \leq d(z)+2 d(y)+d\left(u_{1}\right) \leq 4 n-4,
$$

which is a contradiction.

(ii). Then it is easy to see that

$\vec{a}\left[x_{i}, z\right]+\vec{a}\left[u_{2}, x_{i+1}\right] \leq 1, \quad \vec{a}\left[x_{i}, u_{1}\right]+\vec{a}\left[z, x_{i+1}\right] \leq 1 \quad$ and $\quad \vec{a}\left[x_{i}, u_{2}\right]+\vec{a}\left[u_{1}, x_{i+1}\right] \leq 1$

Hence,

$$
d(z, V(C))+d\left(u_{1}, V(C)\right)+d\left(u_{2}, V(C)\right) \leq 3 n-12 .
$$

Therefore, since $d\left(z,\left\{u_{1}, u_{2}, u_{3}\right\}\right) \leq 4$ and $d\left(u_{1},\left\{z, u_{2}, u_{3}\right\}\right)+d\left(u_{2},\left\{z, u_{1}, u_{3}\right\}\right) \leq 11$, we obtain that $d(z)+d\left(u_{1}\right)+d\left(u_{2}\right) \leq 3 n+3$. This together with $d\left(y,\left\{z, u_{1}, u_{2}\right\}\right)=0$ implies that $d(y) \leq n-3$ and $d(z)+d\left(u_{1}\right)+d\left(u_{2}\right)+3 d(y) \leq 6 n-6$. On the other hand, since $d(z)+d\left(u_{1}\right)+2 d(y) \geq 4 n-3$ and $d(y)+d\left(u_{2}\right) \geq 2 n+1$, we have

$$
6 n-2 \leq d(z)+d\left(u_{1}\right)+3 d(y)+d\left(u_{2}\right) \leq 6 n-6,
$$

which is a contradiction.

(iii). First, notice that $d(y) \leq n-4$. By an argument similar to that in the proof of (ii), we can show that

$$
d\left(z, V\left(C_{n-4}\right)\right)+d\left(u_{1}, V\left(C_{n-4}\right)\right)+d\left(u_{2}, V\left(C_{n-4}\right)\right)+d\left(u_{3}, V\left(C_{n-4}\right)\right) \leq 4 n-16 .
$$

Then, since

$$
d\left(z,\left\{u_{1}, u_{2}, u_{3}\right\}\right)+d\left(u_{1},\left\{z, u_{2}, u_{3}\right\}\right)+d\left(u_{2},\left\{u_{1}, u_{2}, z\right\}\right)+d\left(u_{3},\left\{u_{1}, u_{2}, z\right\}\right) \leq 20,
$$

we have $d(z)+d\left(u_{1}\right)+d\left(u_{2}\right)+d\left(u_{3}\right) \leq 4 n+4$. Besides, from condition $(M)$ and $d(y) \leq n-4$ it follows that

$$
8 n-6 \leq d(z)+4 d(y)+d\left(u_{1}\right)+d\left(u_{2}\right)+d\left(u_{3}\right) \leq 8 n-12,
$$


which is a contradiction. Lemma 5.5 is proved.

Lemma 5.6: Let $C:=x_{1} x_{2} \ldots x_{n-5} x_{1}$ be a cycle of length $n-5$ in $D$ passing through $y$ and avoiding $z$. Let $A=V(D) \backslash V(C))=\left\{z, u_{1}, u_{2}, u_{3}, u_{4}\right\}$. If $d\left(y,\left\{u_{1}, u_{2}\right\}\right)=0$ and $z u_{1} u_{2} z$ is a cycle of length three, then $D$ is Hamiltonian.

Proof. Suppose, on the contrary, that $D$ is not Hamiltohian. By Theorem $4.2, D$ contains no cycle passing through $y$ and $z$. Then $d(y) \leq n-3$. It is easy to see that for all $i \in[1, n-5]$, $\vec{a}\left[x_{i}, z\right]+\vec{a}\left[u_{2}, x_{i+1}\right] \leq 1, \quad \vec{a}\left[x_{i}, u_{1}\right]+\vec{a}\left[z, x_{i+1}\right] \leq 1 \quad$ and $\quad \vec{a}\left[x_{i}, u_{2}\right]+\vec{a}\left[u_{1}, x_{i+1}\right] \leq 1$.

Therefore,

$$
\begin{aligned}
d(z, V(C))+d\left(u_{1}, V(C)\right)+ & d\left(u_{2}, V(C)\right)=\sum_{i=1}^{n-5}\left(\vec{a}\left[x_{i}, z\right]+\vec{a}\left[u_{2}, x_{i+1}\right]+\vec{a}\left[x_{i}, u_{1}\right]+\vec{a}\left[z, x_{i+1}\right]\right. \\
& \left.+\vec{a}\left[x_{i}, u_{2}\right]+\vec{a}\left[u_{1}, x_{i+1}\right]\right) \leq 3 n-15 .
\end{aligned}
$$

Since $d(z, A) \leq 5$ and $d\left(u_{1}, A\right)+d\left(u_{2}, A\right) \leq 15$, it follows that $d(z)+d\left(u_{1}\right)+d\left(u_{2}\right) \leq 3 n+5$. This together with $d(y) \leq n-3, d(y)+d\left(u_{2}\right) \geq 2 n+1$ and condition $(M)$ implies that

$$
6 n-2 \leq d(z)+3 d(y)+d\left(u_{1}\right)+d\left(u_{2}\right) \leq 6 n-4,
$$

which is a contradiction. This proves Lemma 5.6.

Lemma 5.7: Suppose that $C:=x_{1} x_{2} \ldots x_{n-2} x_{1}$ is a cycle of length $n-2$ in $D$ passing through $y$ and avoiding $z$. Let $V(D) \backslash V(C))=\{z, u\}$. If $u \leftrightarrow z$, then $D$ is Hamiltonian.

Proof. Suppose, on the contrary, that $z \leftrightarrow x$ but $D$ is not Hamiltonian. Then, by Lemma 4.5, the vertices $y$ and $x$ are not adjacent. Hence, $d(y) \leq n-2$. Since $D$ is not Hamiltonian, it follows that for every $i \in[1, n-2]$ we have $\vec{a}\left[x_{i}, z\right]+\vec{a}\left[x, x_{i+1}\right] \leq 1$ and $\vec{a}\left[x_{i}, x\right]+$ $\vec{a}\left[z_{0}, x_{i+1}\right] \leq 1$. These imply that $d(z)+d(x) \leq 2 n$. Therefore, by condition $(M)$, we have

$$
4 n-3 \leq d(z)+d(x)+2 d(y) \leq 4 n-4,
$$

which is a contradiction. Lemma 5.7 is proved.

\section{Conclusion}

In the current article, we have examined the Manoussakis conjecture for a digraph to be Hamiltonian. For a digraph with the conditions of the Manoussakis conjecture, a number of theorems and lemmas are proved. Found results may be the first step towards confirming the Manoussakis conjecture.

Added in proof. Recently, using some results of this paper, the author confirmed the Manoussakis conjecture.

\section{Acknowledgement}

The author would like to thank the referees for careful reading and many helpful remarks and suggestions. 


\section{References}

[1] J. Bang-Jensen and G. Gutin, Digraphs: Theory, Algorithms and Applications, SpringerVerlag, London, 2000.

[2] J.-C. Bermond and C. Thomassen, "Cycles in digraphs- A survey", J. Graph Theory, vol. 5, pp.1-43, 1981.

[3] D. Küh and D. Ostus, "A survey on Hamilton cycles in directed graphs", European J. Combin., vol. 33, pp. 750-766, 2012.

[4] M. Meyniel, "Une condition suffisante d'existence d'un circuit Hamiltonien dans un graphe oriente", J. Combinatorial Theory B, vol. 14, pp. 137-147, 1973.

[5] Y. Manoussakis, "Directed Hamiltonian graphs", J. Graph Theory, vol. 16, no. 1, pp. 51-59, 1992.

[6] S. Kh. Darbinyan, "Disproof of a conjecture of Thomassen", Akad. Nauk Armyan. SSR Dokl., vol. 76, no. 2, pp. 51-54, 1983.

[7] S. Kh. Darbinyan, "On Hamiltonian and Hamilton-connected digraphs", Akad. Nauk Armyan. SSR Dokl., vol. 91, no. 1, pp. 3-6, 1990 (for a detailed proof see arXiv: 1801.05166v1, 16 Jan 2018.

[8] B. Ning, "Notes on a conjecture of Manoussakis concerning Hamilton cycles in digraphs, electronic preprint", arXiv:1404.5013v1 [math.CO] 20 Apr 2014, pp. 8.

[9] R. Häggvist and C. Thomassen, "On pancyclic digraphs", J. Combin. Theory B, vol. 20, pp. 20-40, 1976.

[10] J. A. Bondy and C. Thomassen, "A short proof of Meyniel's theorem', Discrete Math., vol. 19, pp. 195-197, 1977.

[11] J. A. Bondy, "Basic Graph Theory: Paths and Circuits", In Handbook of Combinatorics, vol. 1-2, Elsevier, Amsterdam, 1995.

[12] A.Yeo, "How close to regular must a semicomplete multipartite digraph to be secure Hamiltonisity?", Graphs Combin., vol. 15, pp. 481-493, 1999.

[13] S. Kh. Darbinyan, "On pancyclic digraphs", Preprint of the Computing Center of Akademy of Sciences of Armenia, 21 pages, 1979.

[14] S. Kh. Darbinyan, "Pancyclicity of digraphs with the Meyniel condition", Studia Sci. Math. Hungar., vol. 20, no. 1-4, pp. 95-117, 1985. (Ph.D. Thesis, Institute Mathematici Akad. Nauk BSSR, Minsk, 1981).

[15] A. Benhocine, "Pancyclism and Meyniel's conditions", Discrete Math., vol. 58, pp. 113$120,1986$.

[16] F. Harary and L. Moser, "The theory of round robin tournaments", Amer. Math. Monthly, vol. 73, pp. 231-246, 1966.

\section{Submitted 14.12.2018, accepted 18.04.2019.}




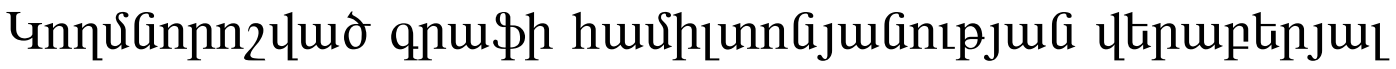

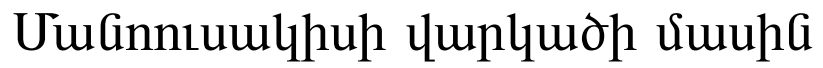

\author{
Uuulki fo. R-unphajug

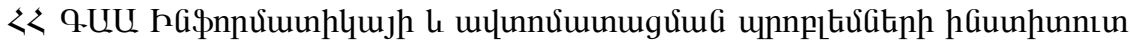 \\ e-mail: samdarbin@ipia.sci.am
}

\section{Uরựnนhnư}

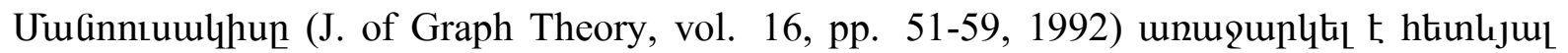
цщициор:

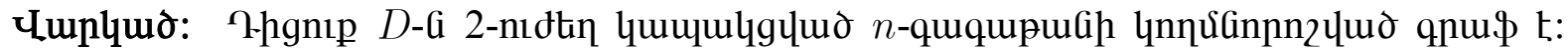

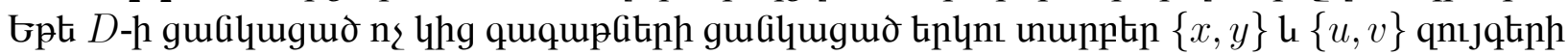

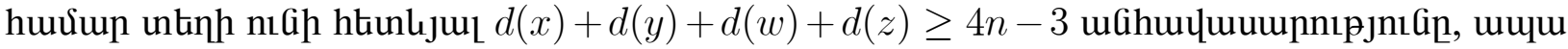

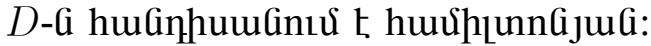

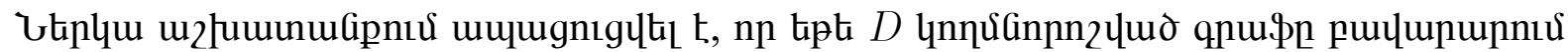

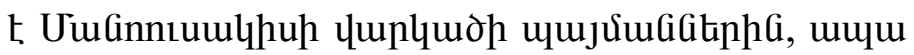

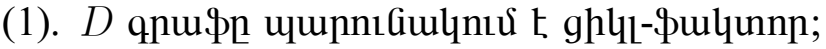

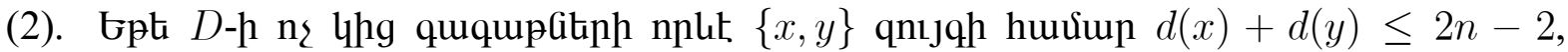

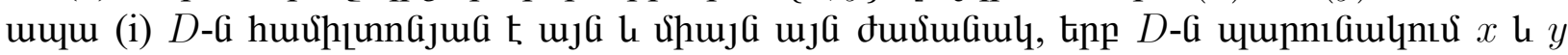

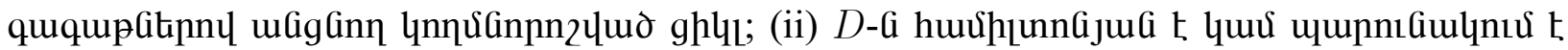

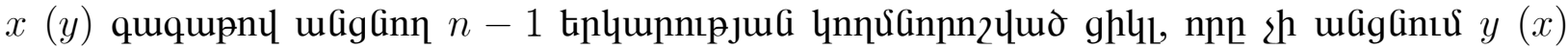

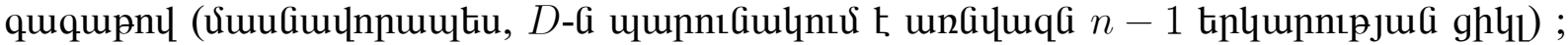

(3). Gpt $D$-h n々 lhg ququpGitph nplit $\{x, y\}$ qnısqh hưuup $d(x)+d(y) \leq 2 n-4$, uщu

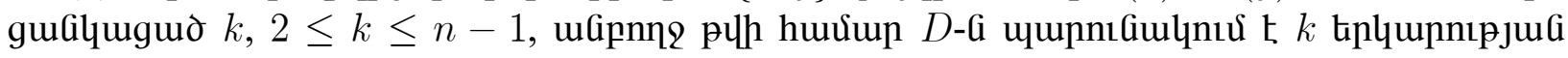

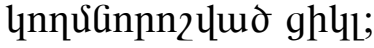

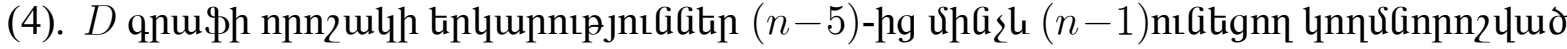

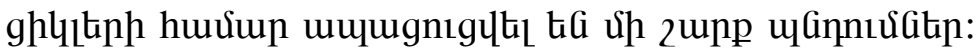

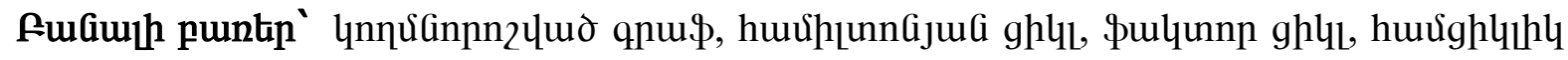
цnпर्ünnzцuд qрuф:

\section{О гипотезе Маноуссакиса о гамильтоновости орграфов}

\author{
Самвел Х. Аарбинян \\ Институт проблем информатики и автоматизации НАН РА \\ e-mail: samdarbin@ipia.sci.am
}

\begin{abstract}
Аннотация
Маноуссакис (J. of Graph Theory, vol. 16, pp. 51-59, 1992) предложил следующую гипотезу.

Гипотеза: Пусть $D$ является 2-сильно связным $n$-вершинным орграфом, в котором для любых различных пар $\{x, y\},\{u, v\}$ несмежных вершин имеет место
\end{abstract}


$d(x)+d(y)+d(w)+d(z) \geq 4 n-3$. Тогда $D$ является гамильтоновым.

В настоящей работе доказано, что если орграф $D$ удовлетворяет условиям гипотеза Маноуссакиса, то

(1). D содержит цикл-фрактор;

(2). Если для некоторой пары несмежных вершин $x$ и $y$ имеет место $d(x)+$ $d(y) \leq 2 n-2$, то имеют место: (i) $D$ является гамильтоновым тогда и только

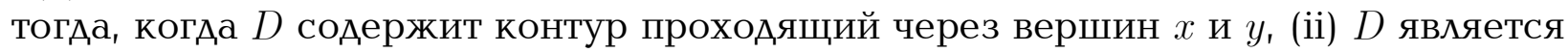
гамильтоновым или содержит контур длины $n-1$, который проходит через вершину $x(y)$ (в частности, $D$ содержит контур длины по крайней мере $n-1$ );

(3). Если Аля некоторой пары несмежных вершин $x$ и $y$ имеет место $d(x)+$ $d(y) \leq 2 n-4$, то $D$ содержит контур любой длины $k, 3 \leq k \leq n-1$;

(4). Аоказаны ряд свойств для контуров длины от $n-5$ до $n-1$.

Ключевые слова: орграф, гамильтоновый цикл, фактор цикл, панциклический орграф. 